\title{
Automatic Alignment of Cranial CT Examinations to the Anterior Commissure/Posterior Commissure (ACPC) Reference Plane for Reliable Interpretation and Quality Assurance
}

\author{
Automatische Ausrichtung kranialer CTs an der Commissura \\ anterior/Commissura-posterior (ACPC)-Referenzebene \\ zur zuverlässigen Bildinterpretation und Qualitätssicherung
}

Authors

Christian Rubbert, Bernd Turowski, Julian Caspers

\section{Affiliation}

University Dusseldorf, Medical Faculty, Department of Diagnostic and Interventional Radiology, D-40225 Dusseldorf, Germany

Key words

multidetector computed tomography, neuroimaging, software, rotation, follow-up studies

received 05.09.2019

accepted 20.04.2020

published online 09.06 .2020

\section{Bibliography}

Fortschr Röntgenstr 2021; 193: 61-67

DOI 10.1055/a-1167-8368

ISSN 1438-9029

(C) 2020. Thieme. All rights reserved.

Georg Thieme Verlag KG, Rüdigerstraße 14,

70469 Stuttgart, Germany

Correspondence

Christian Rubbert

Department of Diagnostic and Interventional Radiology,

University Düsseldorf, Medical Faculty, Moorenstraße 5,

40225 Düsseldorf, Germany

Tel.: ++ 49/211/8117754

Fax: ++49/211/8119487

christian.rubbert@med.uni-duesseldorf.de

\section{ZUSAMMENFASSUNG}

Die Ausrichtung kranialer CTs (cCTs) an einer etablierten Referenzebene unterstützt die Orientierung anhand anatomischer Landmarken und vereinfacht die Verlaufsbeurteilung von Pathologien. Wir haben ein vollautomatisches System als Open Source entwickelt, welches CCTs an der Commissura anterior/Commissura posterior (ACPC) ausrichtet und in das PACS exportiert. Im Ausrichtungsschritt wird das FMRIB Linear Image Registration Tool (FLIRT) mit einem ACPC-orientierten Atlas genutzt. 5mm-Mittelwert-Scheiben mit der obersten soliden Schicht als Ausgangspunkt werden generiert. Zur
Evaluation wurden 301 Trauma-cCTs des CQ500-Datensatzes genutzt. Im visuellen Vergleich mit dem ACPC-orientierten Atlas wurde alle cCTs erfolgreich ausgerichtet. Bildqualität (BQ) und der Aufwand, den Sulcus centralis (SC) zu identifizieren, wurden auf einer Likert Skala eingestuft ( 5 = optimale Bildqualität/auf Anhieb zu identifizierender SC). Die mediane BQ betrug 4 (Spannbreite: $2-4$ ) in den originalen Serien und 5 (4-5) in den ACPC-ausgerichteten Serien $(p<0,0001)$. Der SC war nach fatbACPC einfacher zu identifizieren (Original: 4 (2-5); ACPC: 5 (4-5); $p<0,0001)$. Die mittlere Rotation betrug $|X|=6,4 \pm 5,2^{\circ}\left([-X,+X]=-26,8^{\circ}-24,2^{\circ}\right),|Y|=2,1 \pm$ $1,7^{\circ}\left([-Y,+Y]=-8,7^{\circ}-9,8^{\circ}\right)$ und $|Z|=3,1 \pm 2,4^{\circ}([-Z,+Z]=$ $\left.-14,3^{\circ}-12,5^{\circ}\right)$. Das entwickelte System kann cCTs verlässlich und automatisch an die ACPC-Linie anpassen. Abweichungen von der idealen Ausrichtung könnten zur Qualitätssicherung genutzt werden.

\section{Kernaussagen:}

- fatbACPC richtet kraniale CT Untersuchungen automatisch an der Anterior Commissure/Posterior Commissure Referenzebene aus.

- ACPC-ausgerichtete Bilder erleichtern die Orientierung an anatomischen Landmarken.

- fatbACPC setzt die Bildqualität nicht herab.

- fatbACPC ist robust, vollständig PACS-integrierbar und Open Source: https://github.com/BrainlmAccs

\section{ABSTRACT}

Alignment of cranial CT scans (cCTs) to a common reference plane simplifies anatomical-landmark-based orientation and eases follow-up assessment of intracranial findings. We developed and open sourced a fully automated system, which aligns CCTs to the Anterior Commissure/Posterior Commissure (ACPC) line and exports the results to the PACS. FMRIB's Linear Image Registration Tool (FLIRT) with an ACPC-aligned atlas is used in the alignment step. Five $\mathrm{mm}$ mean slabs are generated with the top non-air slice as the starting point. For evaluation, 301 trauma CCTs from the CQ500 dataset were processed. In visual comparison with the respective ACPCaligned atlas, all were successfully aligned. Image quality (IQ) 
and ease of identification of the central sulcus (CS) were rated on a Likert scale ( 5 = excellent IQ/immediate CS identification). The median IQ was 4 (range: $2-4$ ) in the original series and 5 (range: $4-5)$ in the ACPC-aligned series $(p<0.0001)$. The CS was more easily identified after fatbACPC (original scans: 4 (range: 2-5); ACPC-aligned: 5 (range: 4-5); $\mathrm{p}<0.0001)$. The mean rotation to achieve alignment was $|X|=6.4 \pm 5.2^{\circ}\left([-X,+X]=-26.8^{\circ}-24.2^{\circ}\right),|Y|=2.1 \pm$ $1.7^{\circ}\left([-Y,+Y]=-8.7^{\circ}-9.8^{\circ}\right)$, and $|Z|=3.1 \pm 2.4^{\circ}([-Z,+Z]=$ $\left.-14.3^{\circ}-12.5^{\circ}\right)$. The developed system can robustly and automatically align CCTs to the ACPC line. Degrees of deviation from the ideal alignment could be used for quality assurance.

\section{Key Points:}

- fatbACPC automatically aligns cranial CT scans to the Anterior Commissure/Posterior Commissure plane.

- ACPC-aligned images simplify anatomical-landmark-based orientation.

- fatbACPC does not impact image quality.

- fatbACPC is robust, fully PACS-integrated, and Open Source: https://github.com/BrainlmAccs

\section{Citation Format}

- Rubbert C, Turowski B, Caspers J. Automatic Alignment of Cranial CT Examinations to the Anterior Commissure/ Posterior Commissure (ACPC) Reference Plane for Reliable Interpretation and Quality Assurance. Fortschr Röntgenstr 2021; 193: 61-67

\section{Introduction}

Standardized alignment of cranial cross-section imaging to a common reference plane facilitates accurate and efficient follow-up of intracranial findings [1, 2] and simplifies reliable orientation based on anatomical landmarks [3-5].

In neuroscience and clinical MRI, the Anterior Commissure/ Posterior Commissure (ACPC) line, passing through the apex of the anterior and the inferior edge of the posterior commissure, is the standard axial reference plane for cranial sectional imaging. Talairach and Tournoux originally defined the ACPC line as the reference plane for stereotactic surgery [6]. It allows for reliable anatomic orientation and provides a common basis for standardized stereotactic reporting of findings [7].

The commissures cannot be delineated on CT scout images, and therefore boney or soft-tissue reference landmarks are used to align the scans. To align cranial CT scans to the ACPC line, it was suggested to plan imaging aligned to a line elevated $12^{\circ}$ from the hard palate [8], which was not widely adopted due to irreproducibility and everyday impracticalities. Another approach is using the Tuberculum Sellae/Occipital Protuberance line, parallel to the ACPC line [9]. A drawback to both is the mandatory inclusion of the lenses in order to image the posterior fossa as well. The currently widely used reference plane in $\mathrm{CT}$ is defined by the external auditory canal and roof of the orbits, excluding the lenses, while still including the posterior fossa [10].

In the daily routine, perfect angulation in every scan cannot be achieved, especially in uncooperative patients or when CT scanners with non-tiltable gantries are used. Post-scan reformations are often required and would benefit from automation. In this technical article, we present an open-source project, which will automatically create ACPC-aligned reformations of cranial CT examinations with full Picture Archiving and Communication System (PACS) integration without any end-user interaction. For quality assurance, patient positioning relative to the optimum can be quantified. The approach was validated on a public dataset and has been made available as Open Source (https://github.com/ BrainlmAccs).

\section{Materials and Methods}

A fully automated workflow, which accepts studies sent from a modality or PACS, and processes scans in parallel, was created. It will be referred to as fatbACPC (fully automatic tilting of brain scans to ACPC).

The majority of the scripts in the project were written using the Bourne-again shell (BASH), using the BASH3 boilerplate for consistency, with a small subset using Python. All scripts were extensively commented and released with further documentation on installation and configuration on https://github.com/Brainl $\mathrm{mAccs} /$ fatbACPC. The following is based on fatbACPC v0.3, commit fb52753. The receiving, handling and sending of Digital Imaging and Communications in Medicine (DICOM) files is implemented in https://github.com/Brain/mAccs/BrainSTEM (commit 74d90e8).

\section{DICOM Receiving and Queueing}

DCMTK (OFFIS e. V.) was used to implement a Digital Imaging and Communications in Medicine (DICOM)-receiving backend. The PACS or a modality can be configured to automatically send appropriate series to fatbACPC. These are then fed into a GNU parallelbased queueing system [11] for parallel processing of scans. By default, up to 4 jobs are processed in parallel, which is configurable.

\section{ACPC Alignment}

For further processing, the DICOM images are converted into the Neuroimaging Informatics Technology Initiative (NIfTI) file format using dcm2niix. Robustfov from the FMRIB Software Library v5.0.11 (FSL) [12-14] is used to crop out the neck and lower head on large scan volumes. To clean up the image volume from most voxels not representing brain tissue or skull for registration, all voxels $\leq 0$ Hounsfield Units (HU) are replaced with a voxel value of air $(-1024 \mathrm{HU})$. Then, FMRIB's Linear Image Registration Tool (FLIRT) $[15,16]$ is used to register the cropped image volume with an ACPC-aligned, high-resolution, unsmoothed CT template derived from the Clinical Toolbox for SPM 8/2014 [17], using 12 degrees of freedom (DOF). Representative slices of the tem- 


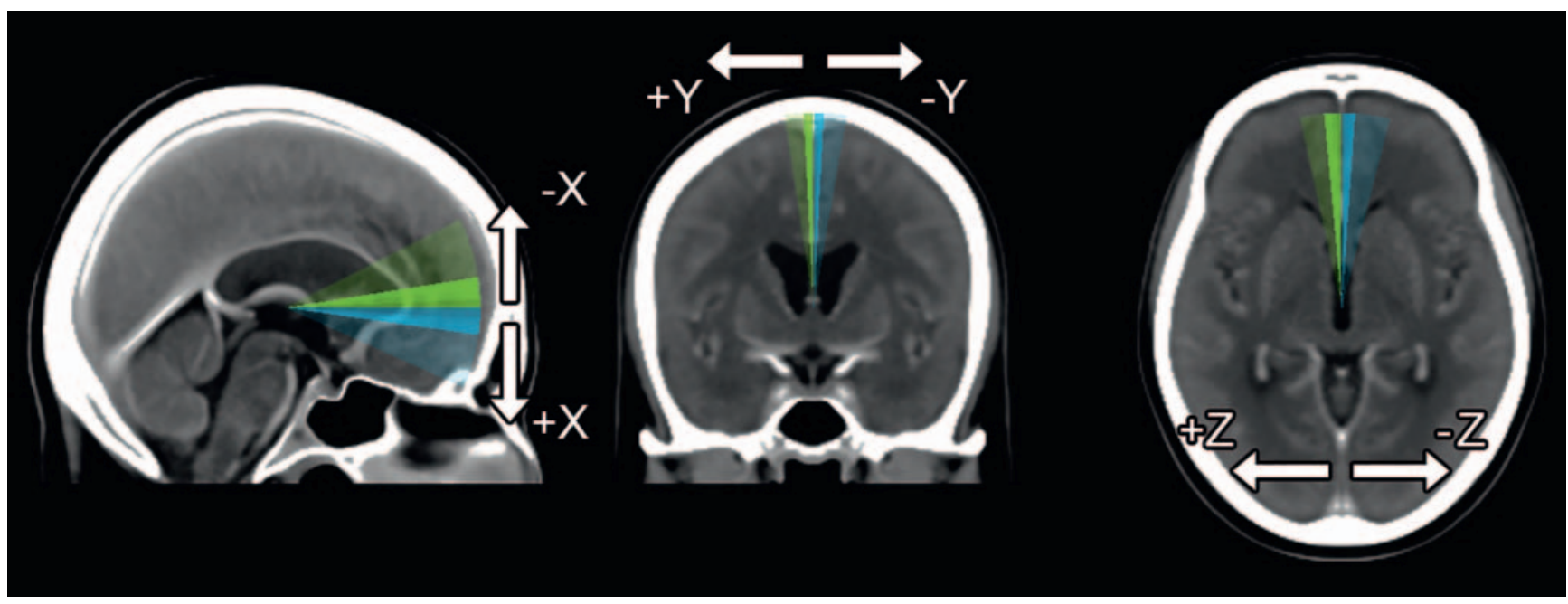

- Fig. 1 Representative slices of the Anterior Commissure/Posterior Commissure (ACPC)-aligned template. The CT template is part of the Clinical Toolbox for SPM 8/2014. Rotation axes after decomposing the rigid translation matrix as well as range (transparent) and interquartile range (solid) of the rotation to achieve ACPC alignment are overlaid.

- Abb. 1 Repräsentative Schichten der Commissura-anterior/Commissura-posterior (ACPC)-ausgerichteten Vorlage. Die CT-Vorlage ist Teil der Clinical Toolbox für SPM 8/2014. Die Rotationsachsen nach Dekomposition der rigiden Translationsmatrix, die Spannweite (transparent) und der Interquartilsabstand (solide) der Rotationen, die für eine ACPC-Ausrichtung notwendig waren, wurden den Schichten überlagert.

plate are shown in $>$ Fig. 1. Using FSL's aff2rigid, a rigid 6-DOFtransformation is approximated from the affine 12-DOF-transformation by aligning the anterior commissure, the ACPC line and the midhemispheric plane in order of decreasing accuracy.

The converted DICOM data might have a larger anterior-posterior and left-right dimension than the ACPC template. Using FLIRT, the dimensions of the registered image will be adapted to the dimensions of the templates. However, after registration, the volume may have been tilted in such an extreme way that the original superior-inferior dimension is too small to contain the whole image volume. To address this issue, axisBounds from fslpy v1.12.0 [18] was used to calculate the z-axis boundaries, and a function was written to center the aligned image in the reference volume by modifying the transformation matrix. The final rigid transformation matrix is then applied to the original image volume to create an ACPC-aligned image volume.

\section{Mean Slabs}

To reduce noise, $5 \mathrm{~mm}$ mean slabs are computed by default for ACPC-aligned cranial CT examinations.

To generate comparable slabs, i. e. always starting at the same position at the vertex of the skull, the minimum voxel value of the central $48 \times 48$ voxels of a slice is extracted. If that value is $\leq 0 \mathrm{HU}$, it is assumed that the slice only contains air. If the minimum voxel value is $>0 \mathrm{HU}$, solid tissue is assumed.

To find the top non-air slice, first the topmost slice is tested, while the bottommost slice is defined as solid. If the topmost slice is denoted "air", the slice half the distance between the last known "air" and "solid" slice is checked and is labeled as the new "air" or "solid" slice depending on its content. The process is repeated until the positions of the "air" and "solid" slices are adjacent, i. e. the topmost solid slice has been found.
Based on the original slice thickness and gap, the thickness of the mean slabs is approximated as close to the default $5 \mathrm{~mm}$ as possible, to avoid another step of interpolation. Mean slabs are calculated in parallel and merged into an image volume. Finally, the height of the voxels in the z-dimension is adjusted to reflect the mean slab thickness.

\section{Conversion to DICOM}

Since the NIfTI format does not contain as much metadata as the original DICOM files, the middle slice of the original image stack is used as the metadata source. The NIfTI file is converted back to DICOM using nifti2dicom v0.4.11 [19]. Information from the reference DICOM slice, such as accession number, window level, content creation and acquisition date, institution and station name, body part, contrast agent (if applicable), and study and protocol name are copied onto the ACPC-aligned DICOM files. These are then exported to the PACS.

\section{Evaluation}

For testing, clinical trauma CT scans from the public CQ500 dataset were processed. The CQ500 dataset was originally acquired in six centers in New Delhi, India, starting late 2017 [20]. From the dataset, only CT scans covering the entire skull, with a slice thickness $\leq 2.5 \mathrm{~mm}$, window center $=40$, and "SOFT" or "STANDARD" kernel were included ( $n=263 / 490$ patients, 301/1,273 series,

- Table 1). Chilamkurthy et al. have used these previously reported-on patients in a deep learning approach to detect abnormalities on cranial trauma CT scans, whereas in this manuscript we used the scans to validate fatbACPC.

The fatbACPC-aligned CQ500 scans were reviewed by a neuroradiologist (CR, 7 years of experience) for successful ACPC alignment. 25 randomly selected pairs of original and ACPC-aligned 
- Table 1 CQ500 - Patient characteristics.

- Tab. 1 Charakteristiken der Patienten im CQ500 Datensatz.

scan mode

\begin{tabular}{|c|c|}
\hline axial (step-and-shoot) & $197(65.4 \%)$ \\
\hline helical & $104(34.6 \%)$ \\
\hline \multicolumn{2}{|l|}{ convolution Kernel } \\
\hline STANDARD & $194(64.5 \%)$ \\
\hline SOFT & $107(35.5 \%)$ \\
\hline \multicolumn{2}{|l|}{ window center } \\
\hline 30 & $3(1 \%)$ \\
\hline 40 & 298 (99\%) \\
\hline \multicolumn{2}{|l|}{ window width } \\
\hline 100 & $186(61.8 \%)$ \\
\hline 300 & $3(1 \%)$ \\
\hline 350 & $1(0.3 \%)$ \\
\hline 400 & $111(36.9 \%)$ \\
\hline \multicolumn{2}{|l|}{ slice thickness (mm) } \\
\hline 0.625 & $297(98.7 \%)$ \\
\hline 1.25 & $4(1.3 \%)$ \\
\hline spacing between slices (mm) & 9.8 (SD 7.7, range $0.625-20.341$ ) \\
\hline \multicolumn{2}{|l|}{ kilovoltage peak (kvp) } \\
\hline 140 & $87(28.9 \%)$ \\
\hline 120 & $214(71.1 \%)$ \\
\hline X-Ray tube current (mAs) & 273 (SD 91.9, range 118-449) \\
\hline gantry detector tilt & $2.8^{\circ}\left(\mathrm{SD} 5.0^{\circ}\right.$, range $\left.0^{\circ}-25.5^{\circ}\right)$ \\
\hline \multicolumn{2}{|l|}{ software versions } \\
\hline cj2_5m3sp4.5 & $25(8.3 \%)$ \\
\hline cj2_5m3sp5.5 & $54(17.9 \%)$ \\
\hline coreload. 81 & $12(4 \%)$ \\
\hline qin. 20 & $112(37.2 \%)$ \\
\hline qin. 3 & $2(0.7 \%)$ \\
\hline sles_hde.132 & $96(31.9 \%)$ \\
\hline \multicolumn{2}{|c|}{ findings (percentage based on $n=263$ patients) } \\
\hline intracerebral hemorrhage & $91(34.6 \%)$ \\
\hline intraventricular hemorrhage & $9(3.4 \%)$ \\
\hline subdural hematoma & $25(9.5 \%)$ \\
\hline extradural hemorrhage & $7(2.7 \%)$ \\
\hline subarachnoid hemorrhage & $20(7.6 \%)$ \\
\hline mass effect & $49(18.6 \%)$ \\
\hline
\end{tabular}

scans were reviewed by two neuroradiologists (CR and JC, also 7 years of experience) for image quality (IQ) and ease of identification of the central sulcus (CS), a key anatomical feature to identify based on anatomical landmarks [3,5]. Pairings and series description were obfuscated, and the images were reviewed in random order on the PACS without any image information or annotations on display. IQ was rated on a Likert scale: $5=$ excellent, optimal IQ, very low image noise, no artifacts, optimal delineation of gray and white matter, 4 = good IQ, low image noise, minimal artifacts, good delineation of gray and white matter; $3=$ moderate IQ, moderate image noise, image artifacts without impairment of image interpretation, slightly reduced delineation of gray and white matter; 2 = poor IQ, high noise, severe artifacts, partial impairment of image interpretation; 1 = nondiagnostic IQ, image interpretation cannot be performed. For the CS, the following Likert scale items were defined: $5=$ immediate identification; 4 = easy identification; 3 =difficult identification; 2 = uncertain identification, 1 = not identifiable. Unweighted Cohen's Kappa was calculated for inter-reader reliability using $R$ v3.5.2 and the Wilcoxon rank sum test was used to find statistically significant differences, set at $p<0.05$, between original and ACPC-aligned series.

Furthermore, the rigid transformation matrices were decomposed into degrees of rotation using the decompose function from fslpy. $X$ denotes rotation in the sagittal, $Y$ in the coronal, and $Z$ in the axial plane ( $\bullet$ Fig. 1 ). The mean, median, standard deviation (SD), range and interquartile range (IQR) of the rotation around each axis were calculated and overlaid over representative slices of the $\mathrm{CT}$ template. 


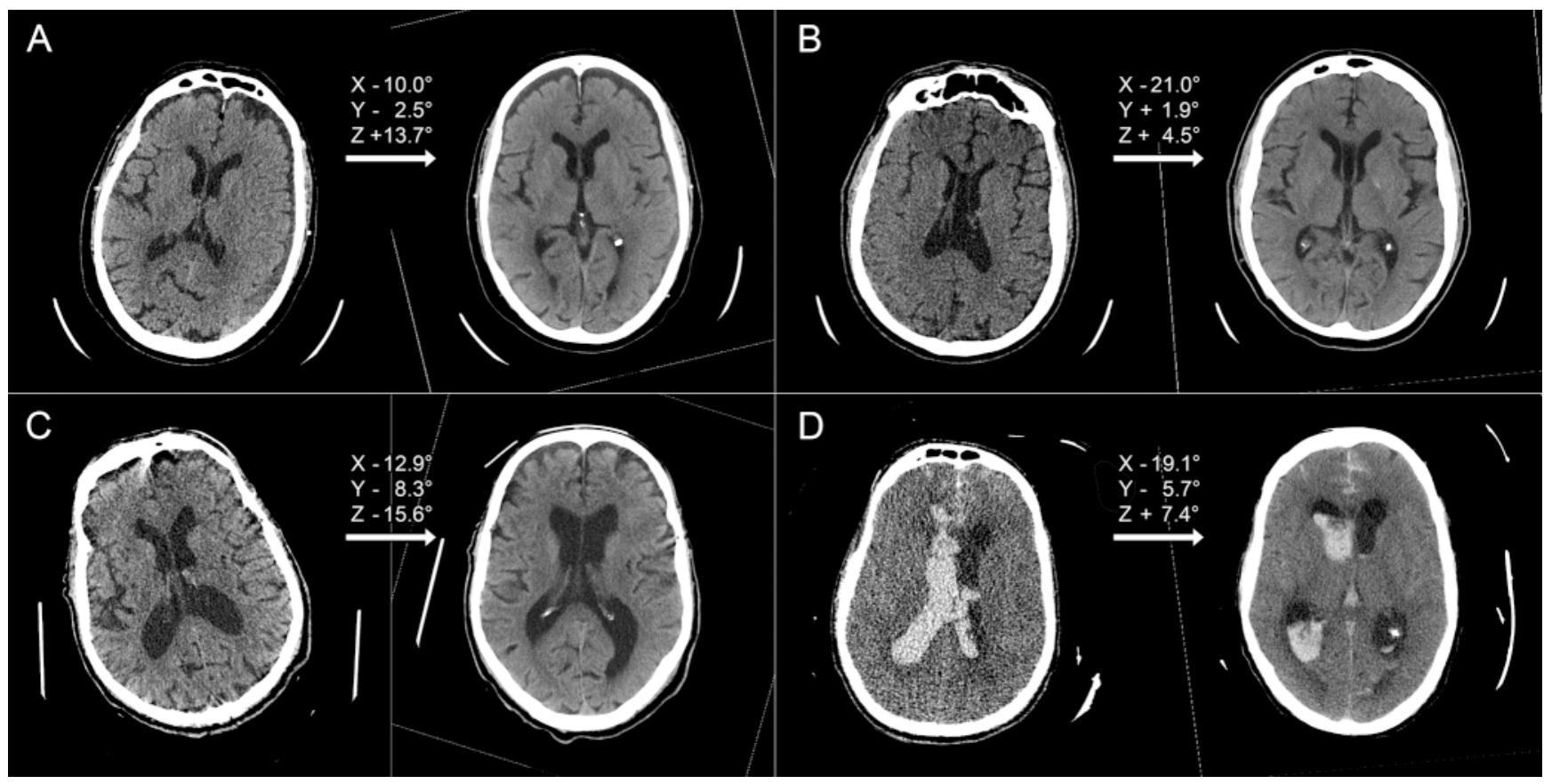

- Fig. 2 Examples of marked rotations performed by fatbACPC. Bounding boxes around the ACPC-aligned scans indicate the amount of rotation, and rotation along each axis to achieve ACPC alignment is shown. A Patient with a small subdural hematoma (SDH) on the left side. Main rotation was performed in the sagittal and axial plane. B Patient with cavum septum pellucidum et vergae. Rotation was mainly performed in the sagittal plane. The right frontal SDH is partially depicted. C Patient without a finding. Rotation was performed along each plane, mainly along the sagittal and axial plane. D Patient with subarachnoid, intraparenchymal and intraventricular hemorrhage. Main rotation was performed along the sagittal and axial plane.

- Abb. 2 Beispiele für CCT-Reformationen mit ausgeprägten Rotationen durch fatbACPC. Die Rahmen um die ACPC-orientierten Schichten geben einen Anhalt für das Ausmaß der jeweiligen Rotation. A Patient mit einem kleinen subduralen Hämatom (SDH) auf der linken Seite. Die Rotation erfolgte überwiegend in der sagittalen und axialen Ebene. B Patient mit Cavum septum pellucidum et vergae. Die Rotation wurde hauptsächlich in der Sagittalebene durchgeführt. Das rechte frontale SDH ist teilweise mitabgebildet. C Patient mit Normalbefund. Die Rotation erfolgte in jeder Ebene, hauptsächlich in der sagittalen und axialen Ebene. D Patient mit subarachnoidaler, intraparenchymaler und intraventrikulärer Blutung. Die Rotation wurde überwiegend in der sagittalen und axialen Ebene durchgeführt.

\section{Results}

Out of 301 series, all (100\%) were successfully ACPC-aligned (examples in - Fig. 2). fatbACPC performed robustly, even in the presence of large mass-effect bleedings or a severe midline shift ( Fig. 3).

The median IQ for the 25 randomly selected original series was rated at 4 (range: $2-5$ ), while the ACPC-aligned counterparts were rated at a median of 5 (range: $4-5$ ) by both readers (Kappa $=0.91$, excellent agreement). IQ was rated one point higher in 20 series by reader \#1 after ACPC alignment, and in 21 series by reader \#2. IQ was two points higher in three series by reader \#1 and four series by reader \#2 after ACPC alignment. The median ease of identification of the CS was rated 4 (range: $2-5$ ) in the original series and 5 (range: 4-5) for the ACPC-aligned series by both readers (Kappa $=0.94$, excellent agreement). The ease of identification was raised by one point on the Likert scale in 17 series by each reader after fatbACPC, and two points in five series by reader \#1 (twice from 2 to 4 , and three times from 3 to 5 ) and 6 series by reader \#2 (twice from 2 to 4 , and four times from 3 to 5). The Wilcoxon rank sum test showed significant differences between the original series and ACPC-aligned series for both features for both readers $(p<0.0001)$.
The mean rotation to achieve alignment was $|X|=5.4^{\circ}$ (SD 5.2), $|Y|=2.1^{\circ}$ (SD 1.7), and $|Z|=3.1^{\circ}$ (SD 2.4), see - Fig. 1. In the axial plane $(\mathrm{Z})$, rotation to the right was performed 146 times $\left(48.5 \%\right.$, median $2.1^{\circ}$, SD $2.3^{\circ}$, range $0-14.3^{\circ}$, IQR $0.9-$ $\left.3.9^{\circ}\right)$, to the left 155 times $\left(51.5 \%\right.$, median $3.0^{\circ}$, SD $2.3^{\circ}$, range 0 $12.5^{\circ}$, IQR $\left.1.5-5.2^{\circ}\right)$. In the coronal plane $(Y)$, rotation to the right was needed in 151 series $\left(50.2 \%\right.$, median $2.1^{\circ}$, SD $1.6^{\circ}$, range $0-9.8^{\circ}$, IQR $\left.0-3.0^{\circ}\right)$, to the left in 150 series $\left(49.8 \%\right.$, median $1.3^{\circ}$, SD $1.8^{\circ}$, range $0-8.7^{\circ}$, IQR $0.6-3.1^{\circ}$ ). In the sagittal plane $(X)$, upward rotation was performed 151 times (50.2\%, median $5.3^{\circ}$, SD $5.8^{\circ}, 0.1-26.9^{\circ}$, IQR $\left.2.4-9.1^{\circ}\right)$ and downwards 150 times (49.8\%, median $4.9^{\circ}$, SD $4.6^{\circ}$, range $0-24.2^{\circ}$, IQR $\left.3.2-8.1^{\circ}\right)$.

The runtime per series was 3.3 minutes (SD 0.8 minutes, median 3.2 minutes, IQR 3.0-3.4 minutes) on a state-of-the-art server (2x Intel E5-2687 W v4 12-Core $3 \mathrm{GHz}$ CPUs), while processing 16 series in parallel.

\section{Discussion}

We developed and open sourced a fully automated workflow, called fatbACPC, which aligns cranial CT scans to the ACPC line without any end-user interaction and exports them to the PACS. 

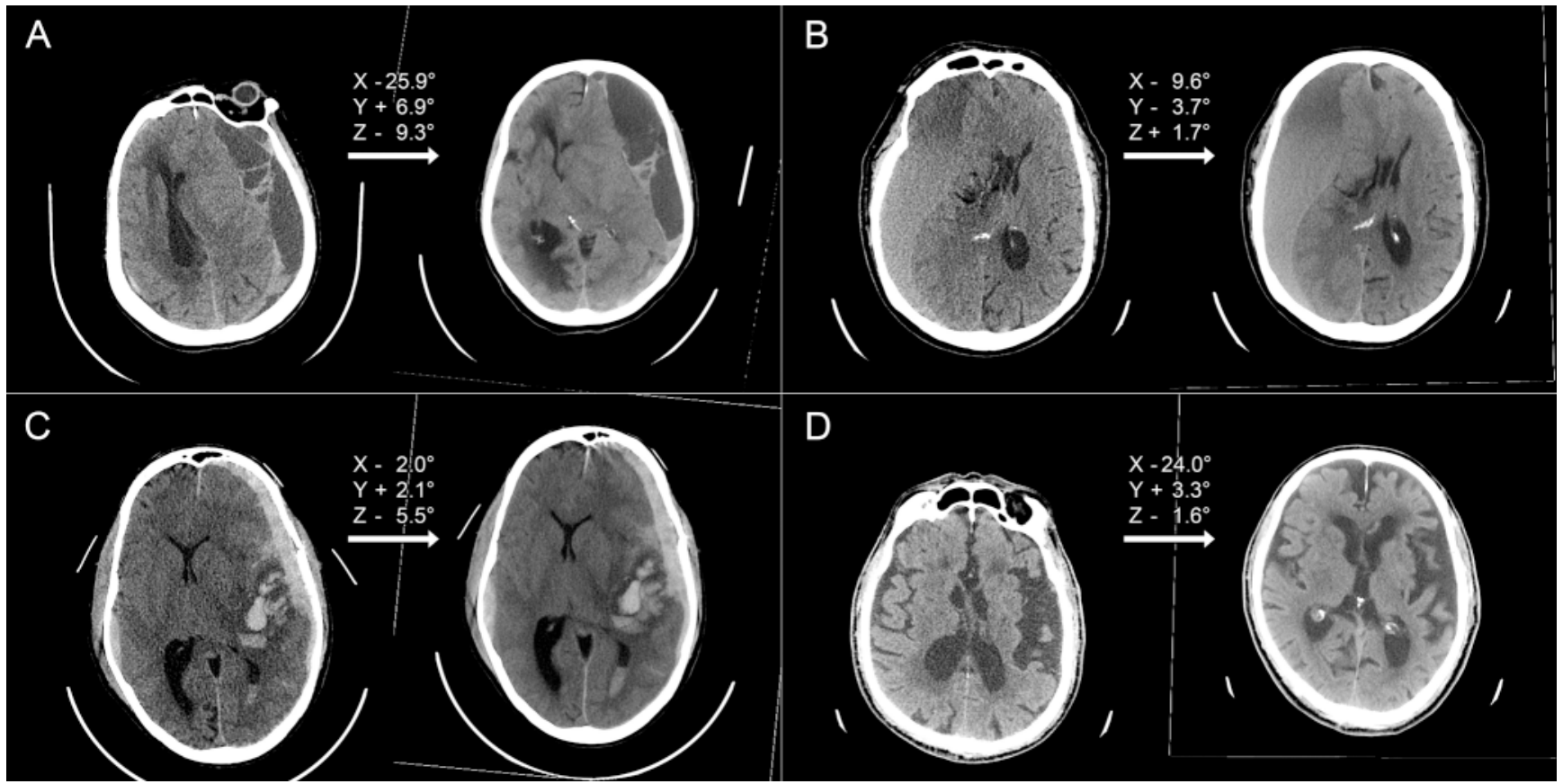

- Fig. 3 Examples of ACPC-aligned examinations with severe pathologies. Bounding boxes around the ACPC-aligned scans indicate the amount of rotation, and rotation along each axis to achieve ACPC-alignment is shown. A Patient with a large septated chronic subdural hematoma (SDH) on the left side. Rotation was performed along each plane, markedly in the sagittal plane. B Patient with a considerable acute SDH on the right side. C Patient with SDH, intraparenchymal and intraventricular hemorrhage. Main Rotation was performed in the axial plane. Notable mass effect and midline-shift is observed in A-C. D Patient with severe left temporal atrophy. Main rotation was performed in the sagittal plane.

- Abb. 3 Beispiele für ACPC-orientierte CCTs mit schweren Pathologien. Die Rahmen um die ACPC-orientierten Schichten geben einen Anhalt für das Ausmaß der jeweiligen Rotation. A Patient mit einem großen, septierten, chronischen subduralen Hämatom (SDH) auf der linken Seite. Die Drehung erfolgte in jeder Ebene, überwiegend in der sagittalen Ebene. B Patient mit einer erheblichen akuten SDH auf der rechten Seite. C Patient mit SDH, intraparenchymaler und intraventrikulärer Blutung. Die Hauptrotation wurde in der axialen Ebene durchgeführt. Die Pathologien in A-C weisen je einen deutlichen raumfordernden Aspekt und eine Mittellinienverlagerung auf. D Patient mit ausgeprägter temporaler Atrophie links. Die Rotation wurde hauptsächlich in der sagittalen Ebene durchgeführt.

Follow-up comparisons are facilitated by creating mean slab stacks always starting with the top non-air slice.

We demonstrated that fatbACPC can very reliably and robustly align cranial CT examinations, even when brain anatomy is severely distorted by pathologies like intracranial hemorrhages. The ACPC line is a useful reference plane as it allows the reliable identification of anatomic landmarks, facilitating accurate anatomical orientation and localization of findings. Our approach can expedite radiologic workflows enormously by automatically and accurately aligning cranial $\mathrm{CT}$ examinations to a common reference plane, which is beneficial for orientation [3-5], interpretation [1], as well as inter-modality comparison [21] and follow-up of intracranial findings [1]. Even if automatic processing were to fail severely, thus not yielding interpretable scans, the source images would still be available for analysis as usual. Earlier works have shown clinical benefits for co-registering prior and current cranial CT scans with a proprietary, non-public software [1]. The study has shown that co-registration of prior and follow-up cranial CT scans "significantly reduces the time needed for comparison and interpretation", non-significantly increases the accuracy of reading, and "tends to decrease intra- and interobserver variability". The interpretation results were changed in $21.9 \%$ of the cases, highlighting the substantial benefit of reliable alignment for fol- low-up evaluation. In our work, we have shown that orientation by anatomical landmarks $[3,5]$ significantly benefits from ACPC alignment. The detection and judgement of findings on ACPCaligned images, as well as the follow-up of findings on ACPCaligned instead of co-registered scans has not been evaluated, yet, and will be subject of future studies. Given the results of Schellingerhout et al., it should be expected that not only co-registration of prior and follow-up scans is beneficial, but also registration of both to a common reference. So far, no system for automatic ACPC alignment of cranial CT scans has been published.

The average processing time is longer than the time required by a radiologist or technician to reformat the images. However, when series are sent automatically by the modality or the PACS immediately after acquisition, ACPC-aligned scans are readily available in time for reporting and the automatic approach may yield more reliably aligned images.

fatbACPC uses publicly available, open-source software at various stages of the workflow, much of which, especially FSL, has been a standard tool in neuroscience for almost two decades, e. g. in the evaluation of functional MRI (fMRI), diffusion tensor imaging, and structural MRI.

Another application of the introduced workflow can be seen in quality assurance. The degrees of deviation from the ideal ACPC 
line could be used in department-wide quality assurance for patient positioning in $\mathrm{CT}$ and sequence planning in MRI. For example, technician training on proper patient positioning or sequence planning can be monitored and optimized by the fatbACPC metrics, and optimal training intervals may be planned based on these evaluations.

A number of conversion steps using different pieces of software are necessary for the conversion of DICOM to NIfTI, alignment, mean slab generation and conversion back to DICOM, which could alter image impression. In the case of the CQ500 dataset, we have shown that IQ is not impaired by applying fatbACPC. On the contrary, IQ improved, which is mainly attributable to the sometimes noisy, medium to low quality of the original series with an axial thickness of $0.625 \mathrm{~mm}$. These benefited from the generation of mean slabs with a thickness of $5 \mathrm{~mm}$. It should be expected that multiplanar reconstruction (MPR) of DICOM data, as carried out, for example, in the PACS, has similar drawbacks and benefits. Our approach could be implemented on the scanner hardware to benefit from access to raw sinogram data, which should yield the best possible IQ. Last but not least, the proposed workflow can also be applied to $3 \mathrm{D}$ MRI images by providing a suitable reference volume, e. g. the ICBM 2009c Nonlinear Symmetric template published by the Neurolmaging \& Surgical Technologies (NIST) Lab.

The scripts and further documentation, i.e., on the software requirements and setup process, have been posted on https://github.com/BrainImAccs. In the spirit of Open Source Software, we cordially invite everyone to contribute.

\section{Conflict of Interest}

The authors declare that they have no conflict of interest.

Acknowledgements

The authors would like to thank Wolf Zinke and Matthew Webster from the FSL mailing list for pointers on mean slab generation.

\section{References}

[1] Schellingerhout D, Lev MH, Bagga RJ et al. Coregistration of head CT comparison studies: assessment of clinical utility. United States: Acad Radiol 2003; 10: 242-248

[2] Forsberg D, Gupta A, Mills C et al. Synchronized navigation of current and prior studies using image registration improves radiologist's efficiency. Int J Comput Assist Radiol Surg 2017; 12: 431-438
[3] Naidich TP, Brightbill TC. Systems for localizing fronto-parietal gyri and sulci on axial CT and MRI. Int J Neuroradiol 1996; 2: 313-338

[4] Naidich TP, Brightbill TC. The intraparietal sulcus: a landmark for localization of pathology on axial CT scans. Int J Neuroradiol 1995; 1: 3-16

[5] Naidich TP, Brightbill TC. The pars marginalis: part I. A "bracket" sign for the central sulcus in axial plane CT and MRI. Int J Neuroradiol 1996; 2: 319

[6] Talairach J, Tournoux P. Co-planar Stereotaxic Atlas of the Human Brain. 1 George Thieme Verlag; 1988

[7] Evans AC, Collins DL, Mills SR et al. 3D statistical neuroanatomical models from 305 MRI volumes. IEEE 1993; 1813-1817

[8] Weiss KL, Storrs ], Weiss JL et al. CT brain prescriptions in Talairach space: a new clinical standard. American Journal of Neuroradiology 2004; 25: 233-237

[9] Kim YI, Ahn KJ, Chung YA et al. A new reference line for the brain CT: the tuberculum sellae-occipital protuberance line is parallel to the anterior/ posterior commissure line. AJNR Am J Neuroradiol 2009; 30: 1704-1708

[10] Yeoman LJ, Howarth L, Britten A et al. Gantry angulation in brain CT: dosage implications, effect on posterior fossa artifacts, and current international practice. Radiology 1992; 184: 113-116

[11] Tange O. GNU Parallel 2018 [Internet]. Zenodo 2018 Mar. Available from: https://doi.org/10.5281/zenodo. 1146014

[12] Woolrich MW, Jbabdi S, Patenaude B et al. Bayesian analysis of neuroimaging data in FSL. Neuroimage 2009; 45 (Suppl. 1): S173-S186

[13] Smith SM, Jenkinson M, Woolrich MW et al. Advances in functional and structural MR image analysis and implementation as FSL. Neuroimage 2004; 23 (Suppl. 1): S208-S219

[14] Jenkinson M, Beckmann CF, Behrens TEJ et al. FSL. Neuroimage 2012; 62: $782-790$

[15] Jenkinson M, Smith S. A global optimisation method for robust affine registration of brain images. Medical Image Analysis 2001; 5: 143-156

[16] Jenkinson M, Bannister P, Brady M et al. Improved optimization for the robust and accurate linear registration and motion correction of brain images. Neuroimage 2002; 17: 825-841

[17] Rorden C, Bonilha L, Fridriksson J et al. Age-specific CT and MRI templates for spatial normalization. Neuroimage 2012; 61: 957-965

[18] McCarthy P, Cottaar M, Webster M et al. fslpy [Internet]. Zenodo 2018. Available from: https://doi.org/10.5281/zenodo.1470750

[19] Domenichelli DE, Arnulfo G. nifti2dicom [Internet]. Zenodo 2018 Sep. Available from: https://doi.org/10.5281/zenodo.1410099

[20] Chilamkurthy S, Ghosh R, Tanamala S et al. Deep learning algorithms for detection of critical findings in head $\mathrm{CT}$ scans: a retrospective study. The Lancet 2018; 392: 2388-2396

[21] Ulin K, Urie MM, Cherlow JM. Results of a multi-institutional benchmark test for cranial CT/MR image registration. Int J Radiat Oncol Biol Phys 2010; 77: 1584-1589 